\title{
Tobacco use disorder and the risk of suicide mortality
}

\author{
Kipling M. Bohnert', ${ }^{1,2}$ Mark A. Ilgen ${ }^{1,2}$, John F. McCarthy ${ }^{1,2}$, Rosalinda V. Ignacio ${ }^{1,2}$, \\ Frederic C. Blow ${ }^{1,2}$ \& Ira R. Katz ${ }^{3}$
}

VA National Serious Mental Illness Treatment Resource and Evaluation Center, Department of Veterans Affairs, Ann Arbor, MI, USA,' Department of Psychiatry, University of Michigan, Ann Arbor, MI, USA² and Department of Veterans Affairs, Veterans Health Administration, Office of Mental Health Services, Washington, DC, USA ${ }^{3}$

\section{ABSTRACT}

Background and aims Tobacco use may be a risk factor for suicide mortality; however, prior research has produced equivocal findings and has been limited by relatively small sample sizes to study the rare event of suicide, as well as a lack of adjustment for other important factors, including psychiatric illness. We estimate the predictive association between tobacco use disorder and the risk of suicide mortality, adjusting for other important variables. Design A prospective cohort study. Setting The United States Veterans Health Administration (VHA). Participants All individuals who received VHA services in fiscal year (FY) 2005 and were alive at the start of FY $2006(n=4863086)$. Measurements Tobacco use disorder was assessed via FYs 2004-05 VHA National Patient Care Database records. The outcome of suicide mortality was assessed during the follow-up interval from the beginning of FY 2006 to the end of FY 2008 using National Death Index records. Findings Of the 4863086 individuals in the study, 4823 died by suicide during the follow-up interval. In the unadjusted model, tobacco use disorder was associated with an increased risk of suicide [hazard ratio $(\mathrm{HR})=1.88 ; 95 \%$ confidence interval $(\mathrm{CI})=1.76,2.02$ ] . After adjustment for model covariates, the association remained statistically significant, although attenuated $(\mathrm{HR}=1.36,95 \% \mathrm{CI}=1.27$, 1.46). Conclusions Tobacco use disorder may confer a modest excess risk of death by suicide. Psychiatric disorders may partially explain the relationship between tobacco use disorder and suicide.

Keywords Cohort, suicide, tobacco use disorder.

Correspondence to: Kipling Bohnert, VA National Serious Mental Illness Treatment Resource and Evaluation Center, Department of Veterans Affairs, Ann Arbor, MI 48109, USA. E-mail: kiplingb@med.umich.edu; kipling.bohnert@va.gov

Submitted 25 April 2013; initial review completed 25 June 2013; final version accepted 1 October 2013

\section{INTRODUCTION}

Suicide is one of the leading causes of death and years of potential life lost in the world. Globally, an estimated 1 million people die by suicide every year [1]. In the United States, suicide ranks among the 10 leading causes of death [2]. In 2009, for example, more than 36000 individuals died by suicide in the United States [2].

Previous epidemiological studies have linked tobacco use and tobacco use disorder (TUD) with an increased risk of suicidal behavior [3-5]. For example, Breslau and colleagues found that daily cigarette smoking predicted subsequent suicidal ideation or non-fatal suicide attempt, even after adjustment for prior depression and substance use disorders [4]. TUD may also be a risk factor for suicide mortality [6-9]. Although prior research has generally linked current tobacco use with an elevated risk of suicide death, several studies have reported a non- significant relationship [10-12]. The equivocal findings may result, in part, from relatively small samples that have been used to study the rare event of suicide. Consequently, a recent meta-analysis pooled 15 cohort studies to further clarify the potential association between tobacco use and the risk of suicide mortality, and found that the risk of death by suicide was 1.81 times greater for current cigarette smokers compared with never smokers [13].

Although meta-analyses are important to examine the consistency of prior findings, they are constrained by the limitations of available studies [14]. Importantly, the studies comprising the Li et al. meta-analysis spanned several decades (1973-2011), and a test of heterogeneity revealed a smaller pooled relative risk estimate for current smokers among studies published after 2000 than studies published in or prior to 2000 [13]. In addition, the study sample sizes ranged from 10934 to 361662 and the 
number of observed suicide events ranged from 34 to 601 [13]. Furthermore, prior studies vary in the extent to which they have included adjustment for potentially important confounding factors when estimating associations between current tobacco use and the risk of suicide death. In particular, psychiatric conditions are correlated with TUD [15,16], and are important risk factors for suicide [17]. Of the 15 studies included in the metaanalysis, only four adjusted for psychiatric illness or stress [10,13,18-20]. Prior assessments of co-occurring psychopathology have ranged from self-reported symptoms of depression and stress to hospital diagnoses of psychiatric disorders. These limitations highlight the need for further inquiry regarding the relationship between current TUD and suicide mortality using more recent data and larger samples, as well as including adjustment for co-occurring psychopathology [17].

In this investigation, we assess the association between current TUD and the risk of suicide death using data from the largest integrated health system in the United States, the Veterans Health Administration (VHA). The study cohort includes all VHA users who obtained care in the system within a single fiscal year (FY), and we assess suicide mortality over the course of a 3-year follow-up interval. This approach ensures a large sample size with sufficient power. In addition, the use of VHA records enables assessment and adjustment for other potentially important factors, including diagnosed psychiatric conditions.

\section{METHODS}

\section{Sample}

We identified all VHA users who received VHA services in FY 2005 and were alive at the beginning of FY 2006. A FY begins on 1 October of the previous calendar year and ends on 30 September of the calendar year (e.g. FY 2005 is 1 October 2004-30 September 2005). A total of 4863086 individuals met study inclusion criteria. The study cohort was then assessed during the 3-year follow-up interval, FYs 2006-2008, for the outcome of suicide mortality. The VA Ann Arbor Institutional Review Board approved project analyses.

\section{Data sources}

Data come from two sources: the VHA National Patient Care Database (NPCD) and the Centers for Disease Control and Prevention's National Death Index (NDI). The NPCD is a national VHA patient medical records database that contains demographic information and information on all VHA in-patient and out-patient encounters, including diagnostic information associated with all VHA clinical contacts $[21,22]$. Diagnoses in the
NPCD are based on clinical assessments and correspond to the International Classification of Diseases, 9th revision, Clinical Modification (ICD-9-CM). The NDI includes information on the date and cause of death for all deaths that occur within the United States [23]. The NDI records are based on death certificates that are filed in state vital statistics offices. Within the NDI, causes of death are classified according to the International Classification of Diseases, 10th revision, Clinical Modification (ICD-10-CM). The NDI is the most sensitive population-based vital status data source in the United States [24]. Using the method described by McCarthy et al. [25] and Ilgen et al. [17,26], all individuals in the study cohort were assessed for VHA service use in FYs 2009 or 2010 (i.e. the two FYs directly following the end of the 3-year study follow-up interval). Those individuals with VHA service use in FYs 2009 or 2010 were assumed to be alive during the study follow-up interval, and were not included in the NDI searches. The NDI searches were then performed for all individuals in the cohort who had received VHA services in FY 2005, were alive at the beginning of FY 2006 and had no record of VHA service use in FYs 2009 or 2010. When multiple potential matches were identified from a search, established procedures identified 'true' matches [27].

\section{Measures}

\section{Suicide mortality}

The outcome of interest in this study was suicide mortality during the 3-year follow-up interval, FYs 2006-2008. Information on dates and causes of death were obtained from NDI data. Suicide was identified using ICD-10-CM codes X60-X84 and Y87.0.

\section{Current TUD}

The focal predictor of interest was current TUD in FYs 2004-2005, as assessed via NPCD records. TUD was identified using the ICD-9-CM diagnostic code 305.1. The diagnostic definition of TUD, as described in the ICD-9 volume I, Modified for US Purposes, is as follows: 'Cases in which tobacco is used to the detriment of a person's health or social functioning or in which there is tobacco dependence [28]'. All TUD diagnoses were based on clinical judgment and recorded by VHA health-care providers during VHA encounters.

\section{Other covariates}

In the adjusted analyses we included other covariates, most notably psychiatric conditions, that might confound the relationship between TUD and the risk of suicide death. The demographic characteristics of age group and 
sex were obtained from FY 2005 NPCD records. Charlson comorbidity score, an established measure that classifies comorbidity according to severity, was used to account for medical comorbidities, and was based on clinical diagnoses from FYs 2004-2005 NPCD records [29]. VHA service connection, categorized as less than $50 \%$ or $50 \%$ or greater, was obtained from FY 2005 NPCD records. Psychiatric diagnoses of substance use disorder, bipolar disorder, depression, other anxiety disorder, posttraumatic stress disorder and schizophrenia were obtained from the FYs 2004-2005 NPCD ICD-9-CM diagnostic records.

\section{Statistical analysis}

All analyses were performed on the closed cohort of 4863086 individuals who used VHA services in FY 2005 and were alive at the start of FY 2006. Descriptive analytical methods were used to examine characteristics of the total cohort and by TUD status. Descriptive analyses also calculated the number and percentage of deaths by suicide during the 3-year follow-up interval among the entire cohort, as well as among those with and without diagnosed TUD. A series of proportional hazards regression models were then used to estimate the association between current TUD and the risk of suicide death, with analyses yielding hazard ratios (HRs) and corresponding 95\% confidence intervals (CIs) as the estimates of association [30]. The first model estimated the unadjusted, bivariate relationship between current TUD and the risk of suicide death. The second model adjusted for age group, sex, Charlson comorbidity score and service connection. The third model was an elaboration of the two previous models, with additional adjustment for co-occurring psychiatric conditions (i.e. substance use disorder, bipolar disorder, depression, other anxiety disorder, post-traumatic stress disorder and schizophrenia). For the analyses, risk time for all individuals in the cohort began on the first day of FY 2006 and ended on the date of suicide or the last day of FY 2008, if alive at that point. Individuals in the cohort who died due to other causes during the follow-up interval were censored at the date of their death. The proportional hazards modeling procedures also took into account the clustered nature of the data (i.e. with patients nested within VHA facilities). All analyses were conducted using SAS version 9.3 .

\section{RESULTS}

Table 1 provides a description of the cohort, overall and by TUD status. Of the 4863086 VHA users, 753368 (15.5\%) had a diagnosis of TUD in FYs 2004-2005. The group with current TUD differed from the group without current TUD on all the socio-demographic and health characteristics presented in the table $(P<0.001)$. Among patients with TUD there were greater percentages of individuals aged 40-49 years and 50-59 years compared with those without TUD. In addition, there was a larger percentage of males in the TUD group. There were also greater percentages of individuals with medical comorbidities (i.e. with a Charlson score of 1 or greater) in the TUD group than in the group without TUD. A greater percentage of those with TUD had VHA service connection of $50 \%$ or greater compared with those without TUD. With respect to co-occurring psychiatric conditions, significantly greater percentages of each psychiatric disorder were observed in the TUD group compared with the group without TUD.

During the follow-up interval 4823 VHA users died by suicide, 1237 in the group with TUD and 3586 in the group without TUD (also shown in Table 1). In addition, 493030 individuals died from other causes during follow-up, and were censored at the date of their death (not shown in the Table). The mean length of follow-up was 1040 days [standard deviation $(\mathrm{SD})=195.1$ ] for the entire cohort, 1038 days $(S D=197.7)$ for those with TUD and 1040 days $(S D=194.7)$ for those without TUD (Table 1). The median length of follow-up was 1096 days (range $=1-1096$ ) for the cohort, and did not differ by TUD group.

Figure 1 displays the plots of the suicide-specific survivor function estimates for the two TUD groups. As shown in the figure, the probability of survivorship was greater for the group without TUD than the group with TUD. During the entire 3-year follow-up interval, a larger percentage of individuals with current TUD died by suicide compared with those without current TUD (0.16 versus $0.09 \%$, respectively; Table 2 ). Results from the proportional hazards regression analyses estimating the prospective association between current TUD and the risk of suicide mortality during follow-up are displayed in Table 2. In the unadjusted, bivariate model, TUD was associated significantly with an increased risk of suicide (HR $=1.88 ; 95 \% \mathrm{CI}=1.76,2.02)$. After adjustment for age group, sex, Charlson comorbidity score and VHA service connection, the HR estimate associated with TUD was modestly attenuated, yet remained statistically significant ( $\mathrm{HR}=1.79 ; 95 \% \mathrm{CI}=1.68,1.91$ ). The association between TUD and the risk of suicide mortality was further diminished in the third model, which adjusted for age group, sex, Charlson score, service connection, substance use disorder, bipolar disorder, depression, other anxiety disorder, post-traumatic stress disorder and schizophrenia; none the less, the estimate remained statistically significant $\quad(\mathrm{HR}=1.36 ; 95 \% \quad \mathrm{CI}=1.27$, 1.46). Furthermore, there was no evidence of violation of the proportional hazards assumption. Namely, the two 
Table 1 Characteristics of the cohort of fiscal year (FY) 2005 Veterans Health Administration users who were alive at the beginning of FY 2006, total and by tobacco use disorder (TUD) status $(n=4863086)$.

\begin{tabular}{|c|c|c|c|c|c|c|}
\hline \multirow[b]{3}{*}{ Characteristic } & \multicolumn{2}{|l|}{ Total } & \multicolumn{4}{|c|}{ Tobacco use disorder (FY 2004/2005) } \\
\hline & \multirow[b]{2}{*}{$n$} & \multirow[b]{2}{*}{$\%$} & \multicolumn{2}{|l|}{ Yes } & \multicolumn{2}{|l|}{ No } \\
\hline & & & $n$ & $\%$ & $n$ & $\%$ \\
\hline Total & 4863086 & 100.0 & 753368 & 100.0 & 4109718 & 100.0 \\
\hline \multicolumn{7}{|l|}{ Age group (years) } \\
\hline $18-29$ & 158967 & 3.3 & 15105 & 2.0 & 143862 & 3.5 \\
\hline $30-39$ & 245266 & 5.0 & 33623 & 4.5 & 211643 & 5.1 \\
\hline $40-49$ & 514083 & 10.6 & 117484 & 15.6 & 396599 & 9.7 \\
\hline $50-59$ & 1161916 & 23.9 & 298868 & 39.7 & 863048 & 21.0 \\
\hline $60-69$ & 955689 & 19.7 & 171313 & 22.7 & 784376 & 19.1 \\
\hline $70-79$ & 1136095 & 23.4 & 92034 & 12.2 & 1044061 & 25.4 \\
\hline $80+$ & 691070 & 14.2 & 24941 & 3.3 & 666129 & 16.2 \\
\hline Male & 4460447 & 91.7 & 712147 & 94.5 & 3748300 & 91.2 \\
\hline \multicolumn{7}{|l|}{ Charlson score } \\
\hline Charlson score $=0$ & 2399120 & 49.3 & 302691 & 40.2 & 2096429 & 51.0 \\
\hline Charlson score $=1$ & 1130024 & 23.2 & 211855 & 28.1 & 918169 & 22.3 \\
\hline Charlson score >1 & 1333942 & 27.4 & 238822 & 31.7 & 1095120 & 26.6 \\
\hline Service connection $\geq 50 \%$ & 618753 & 12.7 & 121759 & 16.2 & 496994 & 12.1 \\
\hline Substance use disorder & 420954 & 8.7 & 206078 & 27.4 & 214876 & 5.2 \\
\hline Bipolar disorder & 109026 & 2.2 & 40037 & 5.3 & 68989 & 1.7 \\
\hline Depression & 853471 & 17.5 & 231278 & 30.7 & 622193 & 15.1 \\
\hline Other anxiety disorder & 345205 & 7.1 & 89274 & 11.8 & 255931 & 6.2 \\
\hline Post-traumatic stress disorder & 359060 & 7.4 & 104657 & 13.9 & 254403 & 6.2 \\
\hline Schizophrenia & 107412 & 2.2 & 40939 & 5.4 & 66473 & 1.6 \\
\hline No. of suicide deaths & 4823 & 0.10 & 1237 & 0.16 & 3586 & 0.09 \\
\hline Mean length of follow-up in days (SD) & 4863086 & $1040(195.1)$ & 753368 & $1038(197.7)$ & 4109718 & $1040(194.7)$ \\
\hline $\begin{array}{l}\text { Median length of follow-up in days } \\
\text { (range) }\end{array}$ & 4863086 & $1096(1-1096)$ & 753368 & $1096(1-1096)$ & 4109718 & $1096(1-1096)$ \\
\hline
\end{tabular}

$\mathrm{SD}=$ standard deviation

survivor curves do not intersect at any time during the follow-up interval (as shown in Fig. 1), and the product term between TUD and event time was not significant $(P=0.8112)$ when it was included in the proportional hazards regression analyses. Also of note, a parallel approach using generalized estimating equations (GEE) logistic regression to estimate the association between TUD and the odds of suicide mortality during the entire 3-year follow-up yielded virtually identical results as the proportional hazards models [i.e. model 1 odds ratio $(\mathrm{OR})=1.88 ; 95 \% \mathrm{CI}=1.76,2.01 ;$ model $2 \mathrm{OR}=1.76$; $95 \% \mathrm{CI}=1.65,1.88 ;$ model $3 \mathrm{OR}=1.34 ; 95 \% \mathrm{CI}=1.25$, $1.44]$.

To understand more clearly the potential independent influence of current TUD on suicide, we conducted a sensitivity analysis among only those individuals in the cohort without a diagnosed psychiatric disorder, i.e. substance use disorder, bipolar disorder, depression, other anxiety disorder, post-traumatic stress disorder and schizophrenia ( $n=3479$ 023). The sensitivity analysis also included adjustment for age group, sex, Charlson score and service connection. The resulting HR estimate for TUD was $1.47(95 \% \mathrm{CI}=1.30,1.65)$. Although somewhat larger, this finding was similar to the HR from the third model in the main analysis.

\section{DISCUSSION}

The findings from the present investigation advance the evidence base regarding the association between current problematic tobacco use and the risk of suicide. In the unadjusted analysis, individuals with current TUD had an estimated 1.88 times greater risk of suicide death during the follow-up interval compared with those without current TUD. After the inclusion of important covariates, the prospective association between current TUD and the risk of suicide death was markedly attenuated (i.e. HR = 1.36); however, the association remained statistically significant.

To our knowledge, the present study is the single largest examination of the potential impact of tobacco use on the risk of death by suicide. The large sample 


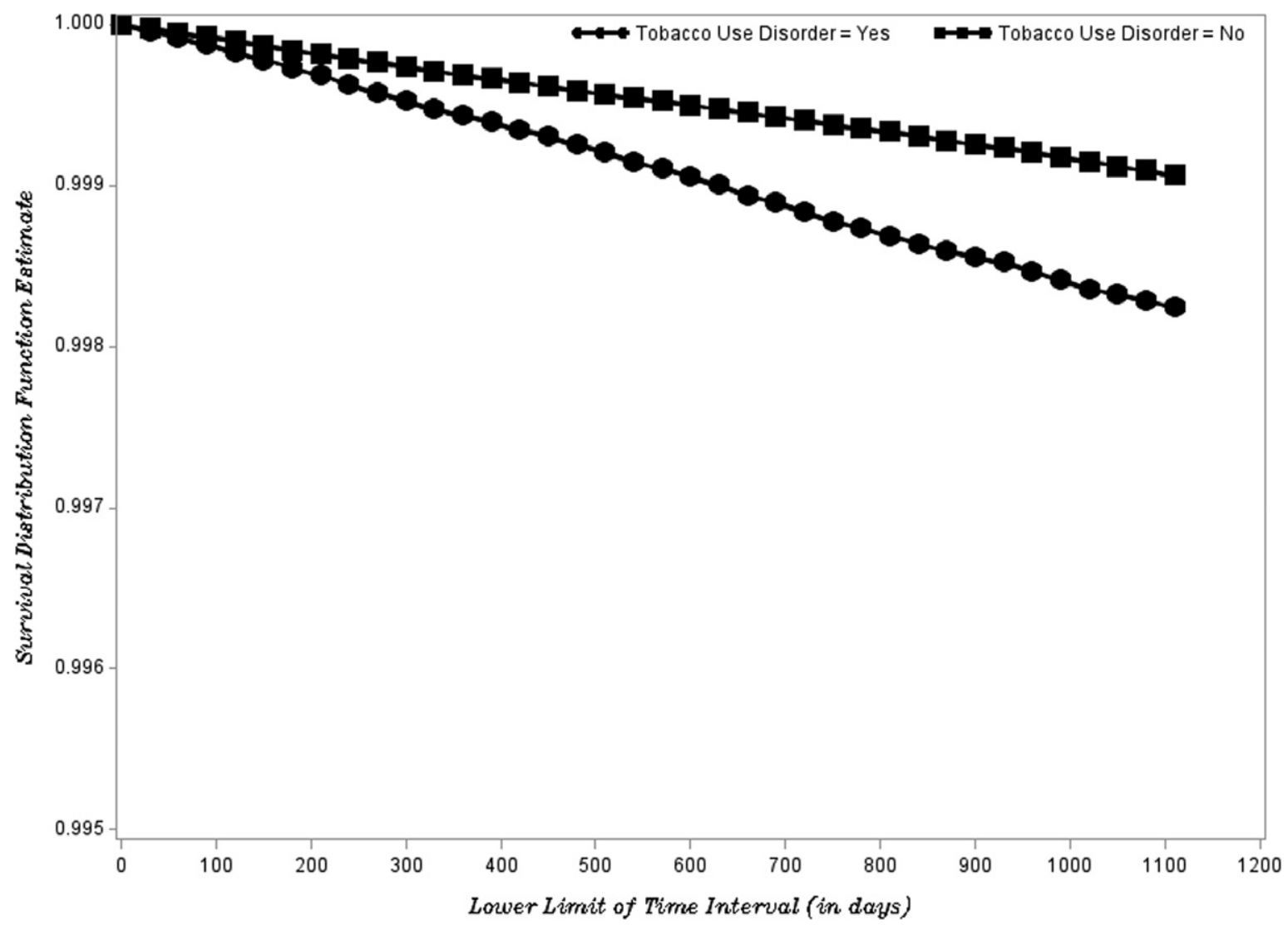

Figure I Suicide-specific survivor function estimates for the two tobacco use disorder (TUD) groups

Table 2 The association between tobacco use disorder (TUD) and risk of death by suicide from fiscal years (FYs) 2006-08 among FY 2005 Veterans Health Administration (VHA) users who were alive at the beginning of FY 2006 ( $n=4863$ 086).

\begin{tabular}{|c|c|c|c|c|c|c|c|c|c|c|c|c|c|c|}
\hline & \multicolumn{2}{|c|}{$\begin{array}{l}\text { Suicide FYs } \\
\text { 2006-2008 }\end{array}$} & \multicolumn{4}{|c|}{ Model $1^{\mathrm{a}}$} & \multicolumn{4}{|c|}{ Model $2^{\mathrm{b}}$} & \multicolumn{4}{|c|}{ Model $3^{\mathrm{c}}$} \\
\hline & $n$ & $\%$ & $H R$ & $95 \% \mathrm{C}$ & & $P$ & $H R$ & $95 \% \mathrm{C}$ & & $P$ & $H R$ & $95 \% \mathrm{C}$ & & $P$ \\
\hline \multicolumn{15}{|l|}{ Tobacco use disorder } \\
\hline Yes $(n=753368)$ & 1237 & 0.16 & 1.88 & 1.76 & 2.02 & $<0.0001$ & 1.79 & 1.68 & 1.91 & $<0.0001$ & 1.36 & 1.27 & 1.46 & $<0.0001$ \\
\hline No $(n=4109718)$ & 3586 & 0.09 & Ref & - & - & & Ref & - & - & & Ref & - & - & \\
\hline
\end{tabular}

$\mathrm{CI}=$ confidence interval; HR = hazard ratio. ${ }^{\mathrm{a}}$ Model 1 is unadjusted. ${ }^{\mathrm{b}}$ Model 2 is adjusted for age group, sex, Charlson score and VHA service connection. ${ }^{c}$ Model 3 is adjusted for age group, sex, Charlson score, VHA service connection, substance use disorder, bipolar disorder, depression, other anxiety disorder, post-traumatic stress disorder and schizophrenia.

size is a major strength of our investigation, and is particularly important when studying a rare outcome such as suicide mortality. We evaluated the hypothesized association among a sample of more than 4.8 million individuals who used VHA services within a single year. During the 3-year follow-up interval, there were 4823 observed suicide deaths. Our sample was approximately three times larger than that of a recent meta-analysis that pooled the findings of 15 prospective cohort studies on the topic [13].

In the published meta-analysis, current smokers had an $81 \%$ greater risk of suicide death than never smokers, a finding comparable with the unadjusted TUD HR estimate from this study [13]. The adjusted estimate from the present investigation is similar to the pooled finding among studies published after 2000 in the meta-analysis [i.e. relative risk $(\mathrm{RR})=1.31] \quad[10,12,13,18,19,31]$, which differed significantly from the larger pooled finding for studies published from 1973 through 2000 [6-9,11,13,20,32-35]. The comparable estimates from the present study and more recent studies may reflect the fact that more recent studies have tended to adjust for other important factors of suicide, including cooccurring psychopathology. Although prior research has 
documented relatively strong associations between psychiatric disorders and the risk of subsequent suicide [17], as well as the common co-occurrence of TUD and psychiatric illness $[15,16]$, few prospective studies examining tobacco use and suicide have considered the potential confounding role of co-occurring psychiatric conditions. Notably, the HR estimate for TUD from our adjusted analysis is most consistent with the pooled RR estimate of 1.40 for current smokers among the four studies that adjusted for mental illness in the published meta-analysis [10,13,18-20].

Taken together, these results suggest that cooccurring psychopathology may partially explain the association between current TUD and the risk of suicide death. Thus, appropriate treatment of comorbid psychiatric conditions may help to lower the risk of suicide among individuals with current TUD and co-occurring psychopathology. None the less, adjustment for cooccurring psychiatric disorders did not completely diminish the association between TUD and suicide, suggesting that current TUD may also be an important independent risk factor for suicide death. Results from the sensitivity analysis lend additional support for the potential independent influence of current TUD on the risk of suicide. Although further research is needed, clinicians' assessment and management of suicidal behavior among patients with current TUD may be important for suicide prevention.

Plausible theories linking current TUD and suicide have been proposed and summarized previously in the literature [3]. One possibility is that the association is spurious, arising via correlation between TUD and another factor (e.g. depression) that is associated with suicide [3]. In our investigation, adjustment for other important factors did not fully attenuate the TUD HR estimate, which may indicate a true association between current TUD and suicide. None the less, we cannot rule out the possibility that an unmeasured, unadjusted factor explains the observed association. Biological and psychological causal mechanisms have also been posited [3]. A predominant theory in the literature emphasizes the role of monamine oxidase (MAO). Namely, current tobacco smoking is associated with lower levels of MAO [36,37], which have also been found to be associated with psychopathology and suicidality $[3,38,39]$. Others have theorized that current tobacco use is related to suicide through its influence on psychological factors, such as mood and impulsivity [3,40]. Further research on these and other potential causal mechanisms will be needed to help clarify the association between current TUD and suicide.

Although the present investigation provides important information on the prospective link between current TUD and the risk of suicide mortality, several potential limitations should be considered. First, the study cohort consisted of all FY 2005 VHA patients who were alive at the beginning of FY 2006. The majority of patients in the VHA are older male Veterans; therefore, study findings may not generalize to patient populations in other settings. Previous epidemiological research has found that older men are at greatest risk for suicide [41], and the potential influence of TUD on suicide may differ in this population subgroup compared with other subgroups. In addition, McCarthy et al. found higher rates of suicide among VHA patients compared with the general US population [25]. Secondly, individuals with current TUD were compared with individuals without current TUD in the study analyses. We were unable to further categorize those without TUD into separate former TUD and never TUD groups. None the less, based on the majority of prior studies that have not found a significant association, former tobacco users and never tobacco users may be equivalent with respect to subsequent suicide risk $[11,12,18]$. Thirdly, although TUD is a marker of tobacco use, it is not a direct measure of tobacco exposure. Moreover, we do not have specific information on the frequency and quantity of patients' tobacco use, such as the number of cigarettes smoked per day, which prevents the examination of a potential dose-response association between tobacco use and the risk of suicide. Fourthly, TUD diagnoses, as well as other psychiatric diagnoses, were derived from VHA administrative records and are based on providers' clinical assessment and judgment. The validity and reliability of these diagnoses are unknown. It is possible that incomplete ascertainment of TUD and/or other psychiatric diagnoses may have influenced the results. Fifthly, as TUD and psychiatric disorders were assessed concurrently, sequence of onset could not be determined. To the extent that there were individuals whose TUD caused their other psychiatric illness, adjustment for psychiatric comorbidity would not be appropriate [42]. Therefore, our adjusted results could be interpreted as a conservative estimate of the effect of TUD on the risk of suicide mortality. Moreover, to help clarify the potential independent role of TUD on the risk of suicide, we conducted a sensitivity analysis among those individuals in the cohort without a diagnosed psychiatric condition. Sixthly, it is possible that we did not adjust adequately for all confounding factors in the final proportional hazards model. For example, in a prior study, the association between tobacco smoking and suicide was explained by heavy alcohol use among tobacco smokers [10]. Although we adjusted for the diagnosis of a substance use disorder, which includes alcohol use disorder, in modeling the association between current TUD and the risk of suicide mortality, inclusion of a measure of level of alcohol use, were it available, rather than substance use disorder may have resulted in a different TUD HR 
estimate. Similarly, future studies may wish to include more detailed measures of socio-economic status when modeling the association between TUD and the risk of suicide mortality.

Despite these limitations, the present study suggests that, independently, current TUD may confer a modest excess risk of death by suicide. Consequently, TUD may be an important factor for health systems and clinicians to incorporate into suicide risk assessment strategies. This research also highlights the importance of considering the potentially confounding influences of co-occurring psychiatric disorders when studying tobacco use and suicide.

\section{Declaration of interests}

None.

\section{Acknowledgements}

This research was supported by the Department of Veterans Affairs (VA) Office of Mental Health Services and the VA Office of Mental Health Operations.

\section{References}

1. World Health Organization. The Global Burden of Disease: 2004 Update. Geneva, Switzerland: World Health Organization; 2008.

2. Kochanek K. D., Xu J., Murphy S. L., Minino A. M., Kung H. Deaths: final data for 2009. Natl Vital Stat Rep 2011; 60: $1-51$.

3. Hughes J. R. Smoking and suicide: a brief overview. Drug Alcohol Depend 2008; 98: 169-78.

4. Breslau N., Schultz L. R., Johnson E. O., Peterson E. L., Davis G. C. Smoking and the risk of suicidal behavior: a prospective study of a community sample. Arch Gen Psychiatry 2005; 62: 328-34.

5. Clarke D. E., Eaton W. W., Petronis K. R., Ko J. Y., Chatterjee A., Anthony J. C. Increased risk of suicidal ideation in smokers and former smokers compared to never smokers: evidence from the Baltimore ECA follow-up study. Suicide Life Threat Behav 2010; 40: 307-18.

6. Friberg L., Cederlof R., Lorich U., Lundman T., De Faire U. Mortality in twins in relation to smoking habits and alcohol problems. Arch Environ Health 1973; 27: 294-304.

7. Hemenway D., Solnick S. J., Colditz G. A. Smoking and suicide among nurses. Am J Public Health 1993; 83: 24951.

8. Miller M., Hemenway D., Bell N. S., Yore M. M., Amoroso P. J. Cigarette smoking and suicide: a prospective study of 300000 male active-duty Army soldiers. Am J Epidemiol 2000; 151: 1060-3.

9. Miller M., Hemenway D., Rimm E. Cigarettes and suicide: a prospective study of 50000 men. Am J Public Health 2000; 90: 768-73.

10. Hemmingsson T., Kriebel D. Smoking at age 18-20 and suicide during 26 years of follow-up-how can the association be explained? Int J Epidemiol 2003; 32: 1000-4.

11. Leistikow B. N., Martin D. C., Samuels S. J. Injury death excesses in smokers: a 1990-95 United States national cohort study. Inj Prev 2000; 6: 277-80.
12. Rebholz C. M., Gu D., Yang W., Chen J., Wu X., Huang J. F. et al. Mortality from suicide and other external cause injuries in China: a prospective cohort study. BMC Public Health 2011; 11: 56.

13. Li D., Yang X., Ge Z., Hao Y., Wang Q., Liu F. et al. Cigarette smoking and risk of completed suicide: a meta-analysis of prospective cohort studies. J Psychiatr Res 2012; 46: $1257-$ 66.

14. Weed D. L. Interpreting epidemiological evidence: how meta-analysis and causal inference methods are related. Int J Epidemiol 2000; 29: 387-90.

15. Grant B. F., Hasin D. S., Chou S. P., Stinson F. S., Dawson D. A. Nicotine dependence and psychiatric disorders in the United States: results from the national epidemiologic survey on alcohol and related conditions. Arch Gen Psychiatry 2004; 61: 1107-15.

16. Centers for Disease Control. Vital signs: current cigarette smoking among adults aged $>/=18$ years with mental illness-United States, 2009-2011. Morb Mortal Wkly Rep 2013; 62: 81-7.

17. Ilgen M. A., Bohnert A. S., Ignacio R. V., McCarthy J. F., Valenstein M. M., Kim H. M. et al. Psychiatric diagnoses and risk of suicide in veterans. Arch Gen Psychiatry 2010; 67: 1152-8.

18. Iwasaki M., Akechi T., Uchitomi Y., Tsugane S. Cigarette smoking and completed suicide among middle-aged men: a population-based cohort study in Japan. Ann Epidemiol 2005; 15: 286-92.

19. Riala K., Alaraisanen A., Taanila A., Hakko H., Timonen M., Rasanen P. Regular daily smoking among 14-year-old adolescents increases the subsequent risk for suicide: the Northern Finland 1966 Birth Cohort Study. J Clin Psychiatry 2007; 68: 775-80.

20. Tanskanen A., Tuomilehto J., Viinamaki H., Vartiainen E., Lehtonen J., Puska P. Smoking and the risk of suicide. Acta Psychiatr Scand 2000; 101: 243-5.

21. Veterans Health Administration. VHA Directive 2009-002, Patient Care Data Capture, Washington, DC. 2009. Available at: http://www.va.gov/vhapublications/ViewPublication .asp?pub_ID=1821 (accessed 12 September 2013) (Archived at http://www.webcitation.org/6KjlYQ1fE on 29 October 2013).

22. Veterans Health Administration Office of Informatics and Analytics. Veterans Health Administration Corporate Databases Monograph. 2011, p. 76. Available at: http://www .virec.research.va.gov/Reports/CDM/CDM-NDS-CY11-ER .pdf (accessed 12 September 2013) (Archived at http:// www.webcitation.org/6Kjlqu45e on 29 October 2013).

23. Centers for Disease Control/National Center for Health Statistics. National Death Index. 2013. Available at: http:// www.cdc.gov/nchs/ndi.htm\#about (accessed 12 September 2013) (Archived at http://www.webcitation.org/ 6KjlvuodO on 29 October 2013).

24. Cowper D. C., Kubal J. D., Maynard C., Hynes D. M. A primer and comparative review of major US mortality databases. Ann Epidemiol 2002; 12: 462-8.

25. McCarthy J. F., Valenstein M., Kim H. M., Ilgen M., Zivin K., Blow F. C. Suicide mortality among patients receiving care in the veterans health administration health system. Am J Epidemiol 2009; 169: 1033-8.

26. Ilgen M. A., Kleinberg F., Ignacio R. V., Bohnert A. S., Valenstein M., McCarthy J. F. et al. Noncancer pain conditions and risk of suicide. JAMA Psychiatry 2013; 70: 692-7. 
27. Sohn M. W., Arnold N., Maynard C., Hynes D. M. Accuracy and completeness of mortality data in the Department of Veterans Affairs. Popul Health Metr 2006; 4: 2. doi: 10.1186/1478-7954-4-2.

28. ICD-9. Volume 1, Modified for US Purposes. 1998. Available at: ftp://ftp.cdc.gov/pub/Health_Statistics/NCHS/ Publications/ICD-9/ucod.txt (accessed 12 September 2013) (Archived at http://www.webcitation.org/6KjmbxinH on 29 October 2013).

29. Charlson M. E., Pompei P., Ales K. L., Mackenzie C. R. A new method of classifying prognostic comorbidity in longitudinal studies: development and validation. J Chronic Dis 1987; 40: $373-83$.

30. Cox D. R. Regression models and life-tables. J R Stat Soc 1972; 34: 187-220.

31. Schneider B., Baumert J., Schneider A., Marten-Mittag B., Meisinger C., Erazo N. et al. The effect of risky alcohol use and smoking on suicide risk: findings from the German MONICA/KORA-Augsburg Cohort Study. Soc Psychiatry Psychiatr Epidemiol 2011; 46: 1127-32.

32. Paffenbarger R. S. Jr, Lee I. M., Leung R. Physical activity and personal characteristics associated with depression and suicide in American college men. Acta Psychiatr Scand Suppl 1994; 377: 16-22.

33. Rantakallio P., Laara E., Koiranen M. A 28 year follow up of mortality among women who smoked during pregnancy. BMJ 1995; 311: 477-80.

34. Smith G. D., Phillips A. N., Neaton J. D. Smoking as 'independent' risk factor for suicide: illustration of an artifact from observational epidemiology? Lancet 1992; 340: 70912.
35. Tverdal A., Thelle D., Stensvold I., Leren P., Bjartveit K. Mortality in relation to smoking history: 13 years' follow-up of 68000 Norwegian men and women 35-49 years. J Clin Epidemiol 1993; 46: 475-87.

36. Whitfield J. B., Pang D., Bucholz K. K., Madden P. A., Heath A. C., Statham D. J. et al. Monoamine oxidase: associations with alcohol dependence, smoking and other measures of psychopathology. Psychol Med 2000; 30: 44354.

37. Fowler J. S., Volkow N. D., Wang G. J., Pappas N., Logan J., Macgregor R. et al. Inhibition of monoamine oxidase B in the brains of smokers. Nature 1996; 379: 733-6.

38. Van Kempen G. M., Notten P., Hengeveld M. W. Repeated measures of platelet MAO activity and 5-HT in a group of suicidal women. Biol Psychiatry 1992; 31: 52930.

39. Lewitzka U., Muller-Oerlinghausen B., Felber W., Brunner J., Hawellek B., Rujescu D. et al. Is MAO-B activity in platelets associated with the occurrence of suicidality and behavioural personality traits in depressed patients? Acta Psychiatr Scand 2008; 117: 41-9.

40. Parrott A. C. Cigarette-derived nicotine is not a medicine. World J Biol Psychiatry 2003; 4: 49-55.

41. Nock M. K., Borges G., Bromet E. J., Cha C. B., Kessler R. C., Lee S. Suicide and suicidal behavior. Epidemiol Rev 2008; 30: 133-54.

42. Leistikow B. Commentary: questionable premises, overadjustment, and a smoking/suicide association in younger adult men. Int J Epidemiol 2003; 32: 1005-6. 